\title{
Deep Learning-Based Text Segmentation in NLP Using Fast Recurrent Neural Network with Bi-LSTM
}

\author{
Vinotheni. $\mathrm{C}^{\mathrm{a}, 1}$ and LakshmanaPandian. $\mathrm{S}^{\mathrm{b}}$ \\ ${ }^{\mathrm{a}, 1}$ Research Scholar, Dept of CSE, Pondicherry Engineering College \\ b Associate Professor, Dept of CSE, Pondicherry Engineering College
}

\begin{abstract}
Segmentation of Text, the undertaking of partitioning a record obsessed by adjoining sections dependent on its semantic design, is a longstanding test in language understanding. Each segment has its applicable significance. Those segments arranged as phrase, text group, point, express or any data unit relying upon the errand of the content examination. This paper proposes the profound learning-based content segmentation strategies in NLP where the content has been portioned utilizing quick tangled neural organization. We propose a bidirectional LSTM prototype where text group embedding is gotten the hang of utilizing fast RNNs and the phrases are fragmented dependent on context-oriented data. This prototype can consequently deal with variable measured setting data and present an enormous new dataset for text segmentation that is naturally divided. Besides, we build up a segmentation prototype dependent on this dataset and show that it sums up well to inconspicuous regular content. We find that albeit the segmentation precision of FRNN with Bi-LSTM segmentation is advanced than some other segmentation techniques. In the proposed framework, every content is resized obsessed by required size, which is straightforwardly exposed to preparation. That is, each resized text has foreordained and these phrases are taken as fragmented content for preparing the neural organization. The outcomes show that the proposed framework yields great segmentation rates which are practically identical to that of segmentation-based plans for manually written content.
\end{abstract}

Keywords.Text segmentation, NLP, bidirectional LSTM, fast-RNN, segmentation accuracy

\section{Introduction}

Text segmentation has been a crucial errand in natural language processing (NLP) that has tended to various degrees of granularity. In this way, segmentation phase is a fundamental advance for preparing thosedialects. At the cognizant section it is known as subject segmentation. Point segmentation is considered as a coarser level, text segmentation by and large alludes to breaking a record obsessed by a grouping of topically pre-essential to help various downstream NLP applications including text rundown and entry recovery.

\footnotetext{
${ }^{1}$ Vinotheni C, Research Scholar, Dept of CSE, Pondicherry Engineering College, India Email: vinotheni95@gmail.com
} 
At a better level, text segmentation alludes to breaking each text group obsessed by the grouping of elementary discourse units (EDUs), regularly known as EDU segmentation [1]. The techniques regularly utilized in customary segmentation of phrases incorporate Maximum Entropy (ME) Markov Prototype, Conditional Random Field (CRF) and Hidden Markov Prototype [2].

The advancement of profound learning and phrase installing innovation types neural networks (NN) a famous decision for characteristic semantic handling significantly diminishes the outstanding burden of highlight designing, and an everincreasing number of researchers apply $\mathrm{NN}$ to tackle segmentation of phrase [3]. The qualities and the construction of the information are gotten through a staggered prototype [4]. The NN has utilized in direct utilization of NN for segmentation of phrase, a Gated Recursive Neural Network (GRNN) with a versatile door and a Long Short-Term Memory Neural Networks (LSTM) usage in segmentation of phases highlighted. In any case, a huge scope profound learning network sets aside an extended effort to track, as well as is developed by a mind-boggling prototype, which needs progress help from PC equipment [5]. The rest of the article is coordinated as the Subdivision 2 is the existing work, Area 3 as the proposed approach, test assessment and results in Section 4 and Section 5 gives future work.

\section{Related Work}

Neural organizations have been broadly utilized for NLP undertakings. The intricacy of archive segmentation rises essentially when considering records which contain text and illustrations; numerous calculations vacillate earnestly as realistic substance are misclassified as text [6]. In this manner text and realistic skewness don't represent a danger to its exhibition, where numerous different methodologies gather huge blunders during archive parsing because of skewness issues [7]. Similarly, the suggested methodology parts a report as three areas (text, reasonable, and establishment), which is a tolerably durable assignment than the division of booklets as two sections (phrase and establishment) [8]. The suggested methodology has been taken a stab at chronicles accumulated from a public database [9], and a division accuracy extent of $84 \% 89 \%$ is obtained for all reports; inducing that no outrageous for any of the individual records with this made division structure [10].

On the opposite side, it has appeared in numerous application spaces that profound learning approaches can give preferred outcomes over hand tailored calculations. For the content line identification issue, the lone works utilizing profound neural organizations are the various commitments of Zayene et al. [11] which propose a blend of Multi-Dimensional Long Short-Term Memory (MDLSTM) neural organization joined with convolutional levels to anticipate a jumping box around the line. In semantic segmentation, one ongoing intriguing technique is the Fully Convolutional Network [2] (FCN), whose thick levels have been eliminated, making them ready to deal with pictures from variable size [12]. The thought behind complete convolutional network functions, where the encoder compares to the CNN without thick levels, and the decoder is an extra part utilized to assemble a yield with a similar goal as the info [13]. Islam et al. [14] proposed a strategy for segmentation in records utilizing RNNs, Badjatiya et al. [15] endeavor familiar with a lucidness work utilizing the halfway requesting relations.[16-18] 


\section{Proposed Methodology}
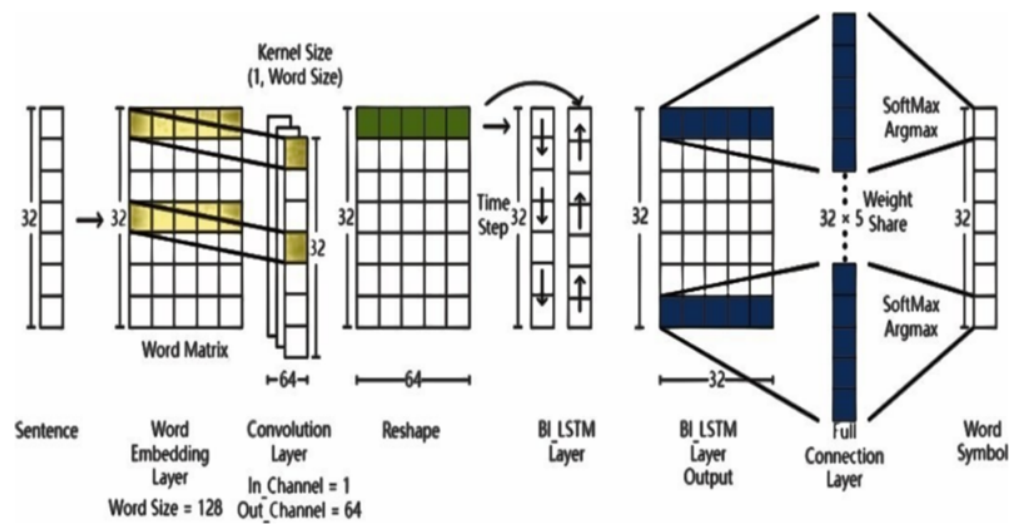

Figure 1. FRNN+ BiLSTM prototype

The underneath figure 1 shows the usage engineering of the proposed neural organization. The FRNN + BiLSTM prototype is suggested to manage division of phrase in this article. In depiction 1, from left to right: (1) Place the entire text group obsessed by the prototype and play out the Inserting taking care of. (2) The yield of the movement (1) is put obsessed by the FRNN system for phrase vector division. (3) Utilize the Bi LSTM association to get setting affiliations. The method uses a fourphase picture technique in which every character about to one of the four pictures, specifically SBME (single phrase, start phrase, focus phrase and end phrase) showing how the phrases are isolated.

\subsection{Labelling Method}

The data collection in this article is shaped through a ton of data in different regions, for instance, books, news data, micro-blog, BBS, and thing appraisals. The method uses a four-phrase picture technique in which every character thinks about one of the four pictures, specifically SBME showing how the phrases are isolated.

\subsection{Embedding Level}

In NLP errands, changing over content obsessed by computerized portrayal is a crucial interaction. This article uses One-Hot encrypting method as well as aides it obsessed by a low-dimensional space using the Entrenching level, which handles the issue of sparse structure and gives the limits a tremendous training space. In this level, the grid cross section An is $32 * \mathrm{~d}$, the lattice A has everything considered 32 lines which means the text group length and the $d$ is the segment of the phrase vector. After the Entrenching level, every text group is changed over obsessed by a grid as the commitment of the fundamental FRNN to get the novel areas. 


\subsection{FRNN Level}

FRNN plans to anticipate the name of the current timestamp with the logical data of past time stamps. Each intermittent level is made by $4 \mathrm{RNN}$ joined at once and to overall longitudinal development of the data. Specifically, we accept as a data an image (or else component guide of the past level) $\mathrm{X}$ of segments $x \in R, H \times W \times C$, where $\mathrm{H}, \mathrm{W}$ and $\mathrm{C}$ are independently the height, width and number of areas (or features) and we divided it obsessed by $I \times J$ areas $p i, j \in R, H p \times W p \times C$. We then clear vertically a first time with two FRNN $f \downarrow$ and $\uparrow$, with $\mathrm{U}$ intermittent units each, that drop top down and base up separately. At each step, each FRNN scrutinizes the accompanying non covering area pi,j and considering its previous state, releases an estimate $o \star i, j$ and revives its state $\mathrm{z} \star \mathrm{i}, \mathrm{j}$ :

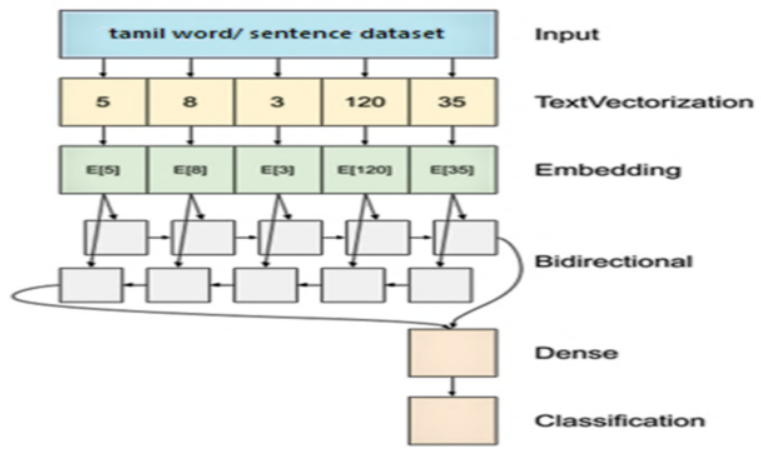

Figure 2. Proposed FRNN architecture

When two vertical FRNN have arranged the whole data X, we interface their estimates $o \downarrow i, j$ and $o \uparrow i, j$ to procure a composite part map Ol whose segments $o l i, j \in R 2 U$ as the commencement of a component locator at the territory $(\mathrm{I}, \mathrm{j})$ with respect to all the areas in the $\mathrm{j}$-th segment of the data. In the wake of getting the associated part map Ol, we clear all its lines two or three new FRNN, $f \rightarrow$ and $f \leftarrow$. With a relative yet specular framework as the one depicted already, we keep scrutinizing one-part oli, $j$ at every movement, to get a connected component $\operatorname{map} O \leftrightarrow$ $=h \leftrightarrow i, j j=1 \ldots J i=1 \ldots I$, before long with $o \leftrightarrow i, j \in R 2 U$. Each segment $0 \leftrightarrow \mathrm{i}, \mathrm{j}$ of this level redundant sublevel addresses the features of one of the data picture areas pi,j with important information.

\subsection{BiLSTM Level}

The level 3 is the BiLSTM level, as well as the novel component vector obtained from the past level data. Differentiated and the standard BiLSTM association, the phrase vector used as data. The prototype count uses the multi-dimensional segment of the phrase vector as data. The BiLSTM prototype is an enhanced LSTM prototype created by RNN prototype with a collaborative period estimation supplementary to the prototype. 


\section{Results and discussion}

\subsection{Dataset}

We evaluate our method on the WIKI-727 test set, Choi synthetic dataset and the two small Wikipedia datasets. We introduce WIKI-50, a set of 50 randomly sampled test documents from WIK-I727K. We use WIK-I50 to evaluate systems that are too slow to evaluate on the entire test set.

\subsection{Performance Analysis}

The performance is based on the evaluation of other parameters such as precision, accuracy in equation 1 defined as number of correctly predicted values to total number of predictions, F1-Score in equation 2 ratios between average mean recall and precision and recall in equation 3 defined as correctly predicted value to total prediction value.

$$
\begin{gathered}
\text { Accuracy }=\frac{T P+T N}{T P+T N+F P+F N} \\
\text { Recall }=\frac{T P}{T P+F N} \\
F 1_{\text {Score }}=2 * \frac{\text { Precision } * \text { Recall }}{\text { Precision }+ \text { Recall }}
\end{gathered}
$$

Table 1. Parametric analysis for proposed technique

\begin{tabular}{ccccc}
\hline Data & Accuracy & Precision & Recall & F-Score \\
\hline 1 & 87.4 & 85.1 & 82.7 & 87.3 \\
2 & 88.6 & 86.2 & 91.3 & 88.3 \\
3 & 90.3 & 89.4 & 89.6 & 90.7 \\
4 & 91.6 & 90 & 92.4 & 92.2 \\
5 & 92 & 93.7 & 92.6 & 91.3 \\
\hline
\end{tabular}

The performance results communicated that preparation exactness slowly increments as quantity of phrases sectioned increment. This is on the grounds that each phrase learns a prototype for the information got by it, and the quantity of phrases increment, quantity of prototypes got by every hub gets lesser and hence forth preparing the prototype for numerous ages overfit the examples and subsequently preparing for precision increments. In table 1 qualities accomplished are introduced. 


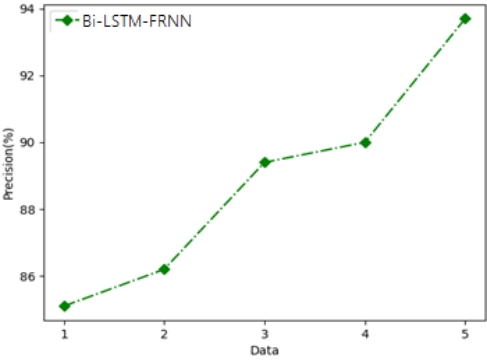

Figure 3. Precision of Research architecture

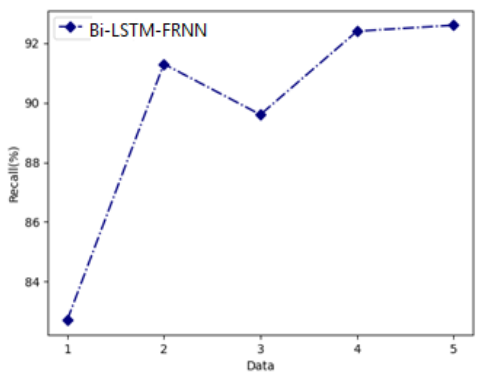

Figure 4. Recall of Research architecture

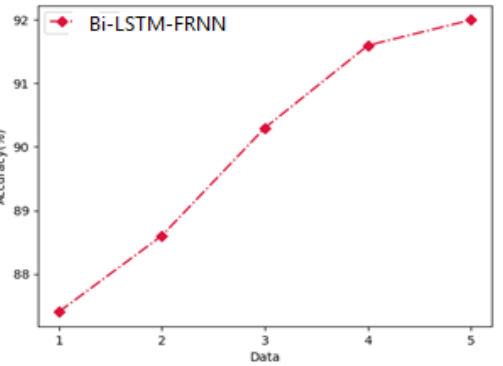

Figure 4. Accuracy of Research architecture

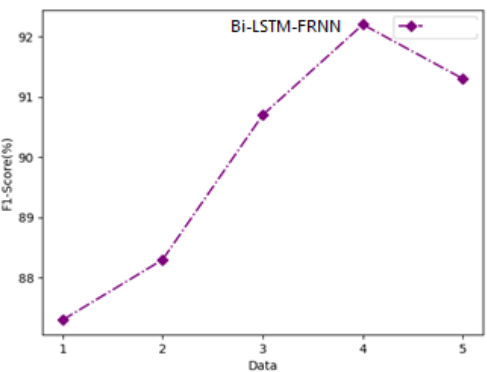

Figure 5. F-Score of Research architecture

The figure 3,4,5,6 shows the precision, accuracy, Recall and F-Score for BiLSTM-FRNN technique. The table 1 shows the variations of values with accuracy and precision for proposed technique. True to form, test exactness diminishes with higher number of hubs in light of the fact that every hub gets a more modest subset of preparing information and thus can't sum up the prototype. Another perception is that outfit adapting consistently gives much preferable precision over the without gathering case (best or normal).

\section{Conclusion}

The exploration works utilized BiLSTM-FRNN for sectioning the content. This paper has introduced a profound learning approach for Text segmentation. To begin with, the phrase information in an entire text group is sectioned and recombined utilizing the FRNN organization; at that point, the recombined fragment is joined in BiLSTM organization; at long last, each expression of the entire text group which is portioned gets relating segmentation of each phrase as the ensuing yield. The prototype finishes the segmentation of phrase tasks productively. The union speed and exactness are raised. It uses not just the benefits of highlight extraction of FRNN organization, yet in addition the upsides of BiLSTM network in planning. Additionally, it is demonstrated that fragmented archives of similar size as the info reports can be acknowledged by reproducing the prepared organization with tests extricated utilizing the covering examining approach. 


\section{References}

[1] Xiong, Ying, et al. A fine-grained Chinese segmentation of phrase and part-of-speech tagging corpus for clinical text. BMC and decision making19.2 (2019): 179-184.

[2] Luo. Segment convolutional neural networks for classifying relations in clinical notes. Journal of American Medical Informatics Association 25.1 (2018): 93-98.

[3] Zagoris K, Chatzichristofis, Papamarkos N. Text localization using standard deviation analysis and support vector machines. EURASIP Adv Signal Process 1 (2011): 1-12.

[4] Kumar MR, Shetty NN, Pragathi BP. Text Line Segmentation of Handwritten Documents using Clustering Method based on Thresholding Approach. International Journal of Computer Applications (0975-8878) on National Conference on Advanced Computing - NCACC, April 2012, India, 9-12.

[5] Vil'kin AM, Safonov IV, Egorova MA (2013). Algorithm for segmentation of documents based on texture features. Pattern Recognit Image Anal 23(1):153-159.

[6] Qiu, Qinjun, et al . DGeoSegmenter: A dictionary-based Chinese phrase segmenter for the geoscience domain, Computers \& geosciences 121 (2018): 1-11.

[7] Li, Xiaozheng, et al. Intelligent diagnosis with Chinese electronic medical records based on convolutional neural networks. BMC bioinformatics 20.1 (2019): 1-12.

[8] Yu, Chenghai, Shupei Wang, and JiajunGuo. Learning Chinese segmentation of phrase based on bidirectional GRU-CRF and CNN network prototype. International Journal of Technology and Human Interaction (IJTHI) 15.3 (2019): 47-62.

[9] Liu, Junxin, et al. Neural Chinese segmentation. Neurocomputing(2019) 46-54.

[10] Yao, Yushi, and Zheng Huang. Bi-directional LSTM recurrent neural network for Chinese segmentation of phrase. International Conference on Neural Information Processing. Springer, Cham, 2016.

[11] Zayene, Oussama, et al. Multi-dimensional long short-term memory networks for artificial Arabic text recognition in video. IET Computer Vision 12.5 (2018): 710-719.

[12] Qiu, Qinjun, et al. DGeoSegmenter: A dictionary-based Chinese phrase segmenter for the geoscience domain. Computers \& geosciences 121 (2018): 1-11.

[13] Shimada,Daiki, RyunosukeKotani, and Hitoshi Iyatomi. Document classification through imagebased character embedding and wildcard training. 2016 IEEE International Conference on Big Data (Big Data). IEEE, 2016.

[14] Islam, MdSanzidul, et al.Sequence-to-sequence Bangla text group generation with LSTM recurrent neural networks. Procedia Computer Science 152 (2019): 51-58.

[15] Badjatiya, Pinkesh, et al. Attention-based neural text segmentation. European Conference on Information Retrieval. Springer, Cham, 2018.

[16] R. Subha Shini et.al., " Recurrent Neural Network based Text Summarization Techniques by Word Sequence Generation",IEEE International Conference on Inventive Computation Technologies (ICICT), 2021, DOI: 10.1109/ICICT50816.2021.9358764

[17] K. Sabarinathan et.al ., “ Machine Maintenance Using Augmented Reality”, 3rd International Conference on Communication and Electronics Systems (ICCES), 2018. (DOI: 10.1109/CESYS.2018.8723900)

[18] B. Aravindh; V.D.Ambeth Kumar; G. Harish; V. Siddartth, “ A novel graphical authentication system for secure banking systems”, IEEE (ICSTM), Pages: $177-183$, 2-4 Aug. 2017, DOI: 10.1109/ICSTM.2017.8089147 